https://doi.org/10.24316/prometeica.v0i18.250

\title{
CAMBIO CLIMÁTICO Y POLÍTICAS PÚBLICAS: UN ABORDAJE DESDE LA TEORÍA SOCIAL DEL RIESGO
}

\author{
CLIMATE CHANGE AND PUBLIC POLICIES: AN APPROACH FROM THE THEORY OF \\ RISK SOCIETY
}

Agustin Roth
(Universidad Nacional de Córdoba / Argentina)
rothagustin@gmail.com

Recibido: $19 / 09 / 2018$

Aprobado: 30/10/2018

\begin{abstract}
RESUMEN
El presente artículo aborda una problemática central de nuestros tiempos: el cambio climático. Este fenómeno produce en el presente consecuencias negativas y dañinas, y se espera que a futuro sus impactos vayan en aumento, amenazando no solamente la economía, el desarrollo y la naturaleza, sino la vida humana misma. El abordaje aquí propuesto será desde la "teoría de la sociedad del riesgo", desarrollada por Beck y Giddens, quienes postulan que la sociedad contemporánea se enfrenta a enormes riesgos y amenazas: riesgos ambientales, nucleares, bélicos, etc.

Dicho panorama, plantea un difícil desafío a las políticas públicas, ya que será fundamental diseñar nuevas políticas estratégicas que estén a la altura del problema, y puedan dar respuesta a los problemas que más preocupan al mundo.

Se propone, por tanto, hacer un recorrido reflexivo por la teoría social del riesgo, y por los desafíos que enfrentan actualmente la política pública y el derecho ambiental para gestionar el cambio climático.
\end{abstract}

Palabras claves: Democracia Ambiental - Percepción de Riesgos - Principio Precautorio Ambiental

\section{ABSTRACT}

The article proposes an analysis of one of the most crucial problems of nowadays: global climate change. This phenomenon is producing negative and severe consequences, and it is expected to continue and to increase in the future, so climate change represents itself a strong threat not only to economy, development and nature, but also to human kind. The approach of the present paper is based on Beck and Gidden's Risk Society Theory, which mainly states that society is constantly facing many risks: environmental ones, nuclear and warlike as well.

The described situation implies a demanding challenge to public policies makers, because new and strategic policies will have to be designed, in order to manage the most urgent problems and threats.

Hence, the paper proposes a wide reflection regarding to risk society theory, and the challenges that climate change represents to public policies and environmental law. 
Keywords: Environmental Democracy — Environmental Precautionary Principle - Risk Perception

\section{Introducción}

En la actualidad una de las mayores amenazas ante las cuales nos enfrentamos es el cambio climático y sus consecuencias negativas sobre la humanidad y el medio ambiente. La comunidad científica en su generalidad, nucleada ante el Grupo Intergubernamental de Expertos sobre el Cambio Climático (IPCC) $)^{1}$, admite que el cambio climático existe, que tiene un determinado componente de causas humanas, y que ya se están produciendo (y seguirán a futuro) alteraciones climáticas que implican desafíos de adaptación y modificación de las formas tradicionales de vida.

Las políticas públicas se enfrentan en el presente al enorme desafío de gestionar adecuada y estratégicamente la amenaza del cambio climático. Pero este fenómeno climático no es igual que otros problemas ambientales o ecológicos: es un problema de escala global, con efectos locales, y genera muchas -demasiadas- incertidumbres sobre sus causas reales, sobre sus efectos actuales y sobre su panorama a futuro.

Son muchos los efectos negativos que ya se están produciendo por el cambio climático, y en nuestro país podemos nombrar, conforme fuera publicado en la "Tercera Comunicación Nacional de la República Argentina a la Convención Marco de las Naciones Unidas sobre el Cambio Climático" 2, a las olas de calor, a las tormentas y precipitaciones extremas, a las inundaciones con mayor frecuencia, el derretimiento de glaciares y del permafrost, sequías e incendios, etc. Y estos daños al medio ambiente y a las personas dejan entrever la incapacidad de las políticas públicas "tradicionales" para hacer frente a la gestión de la crisis ecológica actual y futura.

Sin embargo, el cambio climático global no es la única amenaza actual y futura, sino que en la sociedad moderna y contemporánea se están produciendo y reproduciendo otros riesgos y amenazas de gravedad, como el armamento y energía nucleares, el terrorismo, la contaminación de suelos, agua y aire, la expansión de enfermedades, etc. Vivimos en un mundo convulsionado, cada vez más conectado, tecnificado y cientifizado, donde por todos los frentes aparecen riesgos al ambiente, a la salud y a la forma de producción y trabajo.

En este marco, autores como Ulrich Beck y Anthony Giddens desarrollaron, dos décadas atrás, lo que se conoce como la teoría social del riesgo, que, a grandes rasgos, enuncia que los riesgos (ej. ambientales) representan consecuencias dañinas incalculables e inmanejables, señalando que el avance del conocimiento científico y técnico ya no es capaz de contenerlos. Al contrario de lo que se podría suponer, sostienen que en las sociedades postradicionales "la producción de riesgos es consecuencia de los esfuerzos científicos y políticos por controlarlos o minimizarlos" (Beck, 1998:505), ya que dichos riesgos son un resultado de la intervención humana en el medio ambiente. Ante este panorama, cabe

\footnotetext{
${ }^{1}$ El IPCC fue creado en 1988 para que facilitara evaluaciones integrales del estado de los conocimientos científicos, técnicos y socioeconómicos sobre el cambio climático, sus causas, posibles repercusiones y estrategias de respuesta. Fue creado por el Programa de las Naciones Unidas para el Medio Ambiente (PNUMA) y la Organización Meteorológica Mundial (OMM) en 1988 para ofrecer al mundo una visión científica clara del estado actual de los conocimientos sobre el cambio climático y sus posibles repercusiones medioambientales y socioeconómicas. En el mismo año, la Asamblea General de las Naciones Unidas hizo suya la decisión de la OMM y del PNUMA de crear conjuntamente el IPCC. Consultado en: http://www.ipcc.ch/home_languages_main_spanish.shtml

2 Presentada por nuestro país en noviembre de 2015 (elaboración a cargo de la Secretaría de Ambiente y Desarrollo Sustentable de la Nación hoy jerarquizado en Ministerio homónimo). Estas comunicaciones responden a las obligaciones impuestas por los artículos 4 y 12 de la Convención Marco de las Naciones Unidas sobre el Cambio Climático, normas que obligan a las partes a presentar información sobre el logro de los objetivos de la Convención, y en particular, sobre sus inventarios nacionales de emisiones antropogénicas de GEI (no controladas por el Protocolo de Montreal). Por otra parte, en dicha Comunicación se debe informar sobre los planes de mitigación y adaptación al Cambio Climático. Estas Comunicaciones se erigen en fuentes de datos estadísticos y cualitativos en torno al cambio climático y la postura de nuestro Gobierno Nacional.
} 
preguntarse sobre la real eficacia y estrategia de las clásicas políticas públicas y formas de diseño institucional de los Estados. ¿Son dichas políticas y formas de respuesta suficientes y eficaces para abordar los riesgos a los que se enfrente la humanidad en el presente y hacia el futuro?

Por todo ello, la presente ponencia intentará reflexionar sobre el desafío que el cambio climático significa para la política pública ambiental, teniendo como trasfondo la sociedad del riesgo delineada por Beck y Giddens. En un primer lugar, se abordarán los principales conceptos de la sociedad del riesgo y los caracteres del riesgo; y luego se reflexionará sobre el rol de las políticas públicas y el derecho ambiental frente al gran desafío que implican los riesgos del cambio climático.

\section{La sociedad del riesgo}

En primer lugar, para Beck (2006) la aparición de la sociedad del riesgo implicó una ruptura en la modernidad, diferenciándose esta de la anterior sociedad tradicional decimonónica, también conocida como sociedad industrial. El autor, en su obra, constantemente señala diferencias entre estas dos sociedades, y a continuación se enumera las más llamativas:

- Concepción de la naturaleza: mientras que en la sociedad tradicional o industrial se concibe a la naturaleza como algo externo y escindido de la sociedad, en la actual sociedad del riesgo la naturaleza es algo interior al sistema industrial, "como consecuencia de su transformación técnico-industrial y de su comercialización mundial" (Beck, 2006: 13).

- Idea de progreso: mientras que en la sociedad industrial clásica (S. XIX-XX) había una especie de fe ciega en el automatismo del progreso, en la actualidad los riesgos que el mismo progreso fue generando, hace socavar su fundamento y se lo cuestiona.

- Beck explica que "el concepto de sociedad industrial o de clase (en el sentido más amplio de Marx y Weber) giraba en torno a la cuestión de cómo se puede repartir la riqueza producida socialmente de una manera desigual y al mismo tiempo legítima" (2006: 30). Pero, en la sociedad del riesgo, si bien la idea de clase no desaparece, emerge el problema de la definición y reparto de riesgos producidos, y no solo de las riquezas-miserias.

- En la sociedad tradicional de clases el valor de igualdad es central en su dinámica de desarrollo, mientras que en la sociedad del riesgo el valor más prevaleciente es el de seguridad, y Beck indica que "mientras que la utopía de la igualdad contiene un multitud de fines positivos de los cambios sociales, la utopía de la seguridad es peculiarmente negativa y defensiva: en el fondo, aquí ya no se trata de alcanzar algo 'bueno', sino sólo de evitar lo peor" (2006:69).

- A diferencia de la sociedad tradicional, en la sociedad del riesgo "el pasado pierde la fuerza de determinación para el presente. En su lugar aparece como 'causa' de la vivencia y de la actuación presentes el futuro, es decir, algo no existente, construido, ficticio" (Beck, 2006:48). Todo esto obliga a organizar una acción presente en base a un futuro incalculable y amenazante, donde se erige la problemática de las generaciones futuras.

- Beriain, citando a Luhmarm y Beck, habla de la omnipotencia del peligro, queriendo significar que existe una "angustia de los grupos sociales ante los peligros de la energía nuclear, las guerras y la pobreza no es ninguna evaluación del riesgo, sino que afecta a la comunidad entre la tierra, las plantas, los animales y los seres humanos de tal manera que existe una solidaridad de los seres vivos porque todos estamos «en el mismo barco» y el mar es el mismo para todos, este compartir el mismo Kosmos kairos nos une en la democracia del peligro" (1996: 25).

En segundo lugar, Giddens también da cuenta de ciertas diferencias en la sociedad contemporánea, y postula que "el mundo en el que vimos actualmente no está sometido a un dominio estricto por parte de los humanos [...] Casi al contrario, es un mundo de confusión e incertidumbre" (1998: 13). El autor hace hincapié en que el conocimiento humano y la intervención en la naturaleza, en vez de crear más certeza y seguridades, ha tenido un efecto totalmente contrario, donde se han ido generando 
incertidumbres fabricadas e imprevisibilidades debido justamente a esta intervención y avance del conocimiento.

En relación a la incertidumbre fabricada, Giddens explica que lo que ha cambiado es el origen y el alcance de los riesgos y amenazas, indicando que "el riesgo fabricado es resultado de la intervención humana en las condiciones de la visa social y la naturaleza" (1998:13-14). Podemos afirmar entonces, que los peligros de catástrofes ecológicas no son -simplemente- consecuencias del cambio climático (como algo externo), sino que tienen una relación directa con la intervención del hombre en la naturaleza.

Para comprender aún más a la sociedad del riesgo, vale tener en cuenta la explicación que dan estos autores sobre el porqué del avance de las incertidumbres creadas y los riesgos, y en ese sentido señalan que se debe principalmente a tres factores. El primero, la universalización, donde se transforma el espacio y el tiempo y se la define como acción a la distancia, donde "mi decisión de comprar cierta prenda de vestir tiene repercusiones no sólo en la división internacional del trabajo sino en los ecosistemas terrestres" (Giddens, 1997: 14).

El segundo factor, es la destradicionalización o sociedad post tradicional, donde en realidad las tradiciones no desaparecen, sino que se transforman y se abren a preguntas y debates, a cuestionamientos morales y cosmopolitas.

Por último Giddens señala que "el tercer cambio esencial que influye en las sociedades contemporáneas es la expansión de la capacidad social de reflexión", lo cual genera "la discrepancia entre el conocimiento y el dominio de la situación, un elemento que constituye una fuente esencial de incertidumbre fabricada" (1998: 16). Beck explica que "la modernidad se vuelve reflexiva, es decir, preocupada por sus consecuencias no intencionales, sus riesgos y sus fundamentos" (Beck, Giddens y Lash, 1994: 513).

Es así que ambos autores señalan con agudeza que en la sociedad actual, post tradicional, más conocimiento no significa más control y gestión de un problema ${ }^{3}$, en este caso ambiental. Todo lo contrario: a causa de todo el conocimiento y tecnologías desarrolladas, vivimos en un mundo más contingente y con riesgos incalculables, o en otras palabras, vivimos en un mundo más abierto y desconocido a la vez.

Las diferencias remarcadas con respecto a la sociedad industrial-clásica y "de clases" es lo que va configurando las características de la nueva sociedad del riesgo, donde el futuro amenazante y el reparto de riesgos y responsabilidades aparecen como central.

\section{Caracteres de riesgo}

Para esquematizar y organizar la lectura, se señalan a continuación los caracteres de la noción de "riesgo" más notorios según Beck (2006):

Los riesgos de la sociedad actual no reconocen fronteras ni se limitan a ciertos Estados nacionales, sino que tienen una tendencia globalizadora ${ }^{4}$; tampoco son específicos de una clase social, sino que todos se ven afectados por ellos, incluso los propios productores de riesgos y

\footnotetext{
${ }^{3}$ Beck dice: "Muchos sociólogos (incluyendo a Foucault o Adorno y Horkheimer, teóricos críticos de la escuela de Frankfurt) concibieron la modernidad como una cárcel de conocimiento técnico. (...). Sin embargo, la sociedad de riesgo, al contrario de la imagen que ofrece el término, constituye un mundo mucho más abierto y contingente de lo que podría sugerir cualquier concepción clásica de la sociedad moderna y es así precisamente debido a y no a pesar de todo el conocimiento que hemos generado acerca de nosotros mismos y del entorno material" (1998: 503-504).

4 Beck dice que los riesgos "son un producto global de la maquinaria del progreso industrial y son agudizados sistemáticamente con su desarrollo ulterior" (2006:33).
} 
las clases ricas y poderosas 5 . En este sentido, Beck señala que "objetivamente los riesgos despliegan dentro de su radio de acción y entre los afectados por ellos un efecto igualador" (2006: 52). Sin embargo, el autor aclara en reiteradas oportunidades que muchos riesgos siguen la suerte de las capas o clases sociales, produciéndose, en sus palabras, un "solapamiento entre la sociedad de clases y la sociedad del riesgo [...] las riquezas se acumulan arriba, los riesgos abajo" (2006: 50).

> Los riesgos actuales (por ejemplo las sustancias químicas nocivas para la salud humana presentes en el agua, aire y alimentos) suelen permanecer "invisibles", ya que son imperceptibles a los sentidos de las personas, y en el momento de hacerse conscientes, suelen generar incertezas, desacuerdos y, tristemente, daños irreversibles a la salud.

$>$ Beck, señala con agudeza que "los riesgos se refieren a un futuro que hay que evitar" (2006: 48). Lo que quiere significar el autor con dicha frase, es que los riesgos a los cuales nos enfrentamos no son solamente actuales sino que tienen una "gran cuota" a futuro de peligros y amenazas que si bien no se sabe si se van a producir o no, ni cuándo ni cómo, pero sí generan una reacción en la sociedad que reclama actuar hoy para mitigar y evitar los daños futuros. Este carácter tiene una influencia muy importante en la configuración de las políticas públicas contra el cambio climático.

Son innumerables los casos donde se generan situaciones de riesgos con las características señaladas, pero basta con reflexionar sobre el preocupante caso que alarmó a la ciudad de Córdoba a comienzos del año 2017, cuando a raíz de una inundación, el agua ingresó a la planta de residuos peligrosos "Taym”, y luego fluyó hacia el canal Dique Los Molinos. Automáticamente la sociedad cordobesa se vio presa del riesgo y del miedo de que agua contaminada, tóxica y peligrosa llegara a su casa por la red de agua. A esta inseguridad y catástrofe se le sumó el desconocimiento general sobre la temática de la sanidad del agua, la disputa entre activistas ambientales, expertos científicos, responsables de la planta de tratamiento y los funcionarios políticos. Versiones cruzadas, incertezas, miedo, desconcierto, medios de comunicación, política y ciencia, se vieron todas inmersas en un triste episodio que, en cuestiones de segundos, hizo generar una situación de riesgo grave para miles de personas.

Luego de interrumpirse el suministro de agua, se hicieron análisis científicos y los mismos dieron como resultado que el agua se encontraba "dentro de los parámetros admitidos por la legislación vigente". El temor se fundamentaba por la posible presencia de metales pesados y otros tóxicos en el agua. Y si bien los análisis arrojaron que el agua se encontraba "dentro de los parámetros de la ley", ¿no sería razonable seguir dudando ante un accidente de tal magnitud? ¿En base a qué certeza las leyes establecen parámetros mínimos de contenido de tóxicos en los alimentos o el agua? ¿Tienen en cuenta las leyes la posibilidad de que la sumatoria de "tóxicos en cantidades mínimas e inocuas" provoquen daños en nuestra salud? Este caso, demuestra con firmeza que el conocimiento científico por sí solo no alcanza ya para resolver los problemas, y que los riesgos actuales generan incertidumbre, inseguridades y posibles daños invisibles a las personas.

\section{Cambio climático y políticas públicas}

El cambio climático es un fenómeno mundial sobre el cual existen variadas posiciones, que a su vez, representan prácticas discursivas con todo un telón de fondo representativo de relaciones de podersaber. A fines ilustrativos, tenemos dos grandes posturas: por un lado los negacionistas del fenómeno actualmente liderados por el presidente de los Estados Unidos Donald Trump- (Fresneda, 2016), y por otro lado, a los que admiten que el cambio climático está sucediendo y afectando a la humanidad (postura encabezada por la Organización de las Naciones Unidas y el Grupo Intergubernamental de Expertos sobre el Cambio Climático).

\footnotetext{
${ }^{5}$ Con mayor precisión Beck expresa que "tampoco los ricos y poderosos están seguros ante ellos. Y esto no sólo en tanto que los peligros para la salud, sino también en tanto que peligros para la legitimación, la propiedad y la ganancia" (2016:34).
} 
Nuestro país se enrola mayormente, y ante la Comunidad Internacional, en la postura que admite al fenómeno, ya que en 1993, mediante ley 24.295 fue aprobada la Convención Marco de las Naciones Unidas sobre el Cambio Climático (en adelante, CMNUCC), la cual define en su artículo 1, párrafo 2 al "cambio climático" como un "cambio de clima atribuido directa o indirectamente a la actividad humana que altera la composición de la atmósfera mundial y que se suma a la variabilidad natural del clima observada durante períodos de tiempo comparables" (artículo 1, párrafo 2, CMNUCC). Dicha definición no solo admite la existencia del fenómeno, sino que también atribuye sus causas a las actividades antropogénicas.

Ante este panorama, nuestro país ha venido desarrollando diversas políticas públicas para hacer frente al cambio climático, como por ejemplo y solo por nombrar algunas: el fomento de energías renovables $^{6}$; la creación, en la órbita de la Jefatura de Gabinete de Ministros, del Gabinete Nacional de Cambio Climático ${ }^{7}$; la aprobación del Acuerdo de Paris, celebrado en París (Francia), en el marco de la Conferencia de las Partes $\mathrm{N}^{\circ} 21$ (reuniones anuales en el marco de la CMNUCC) ${ }^{8}$; la creación del Programa Nacional de Escenarios Climáticos", teniendo por funciones las de "llevar a cabo estudios sobre la vulnerabilidad de los sistemas humanos naturales al cambio y la variabilidad climática y sobre su capacidad para adaptarse a los mismos" (artículo 2, resolución 248/2005, Secretaría de Ambiente y Desarrollo Sustentable); etc. Es decir que frente a un fenómeno que es considerado como un problema ambiental, el Estado se encuentra con la necesidad de gestionarlo mediante el dictado y ejecución de variadas políticas públicas ambientales.

\section{La problemática de las políticas públicas ambientales en el marco de la sociedad del riesgo}

Como se indicó en párrafos anteriores, el cambio climático es percibido por nuestro Estado como un riesgo, y consecuentemente se elaboran desde hace décadas diversas políticas públicas ambientales para intentar gestionar y hacer frente a la situación de amenaza.

Beck indica que en la sociedad del riesgo "no está claro qué tipo de política y de instituciones políticas está en condiciones" de dominar el riesgo, surgiendo "un vacío de competencia e institucionalidad políticas, incluso de ideas respecto a cómo enfrentar y gestionar las amenazas a futuro" (2006:68). Giddens, por su parte, indica que a los nuevos riesgos "no se les pueden aplicar viejos remedios; pero tampoco responden a la receta de la Ilustración: a más conocimiento, más dominio" (1998: 14). A su vez, este último señala que los riesgos "expresan una causalidad y temporalidad de tanta amplitud, difusión y extensión que se escapan a los modos ortodoxos de atribución. No sabemos cómo abordar adecuadamente este tipo de amenazas" (1997: 158).

Y precisamente ese es el gran dilema actual: las políticas públicas ambientales que tradicionalmente se vienen aplicando ¿son lo suficientemente adecuadas para gestionar semejante riesgo? Es una pregunta que el futuro sabrá responder, pero permite ilustrar lo complejo que es intentar aplicar políticas para solucionar un problema del cual ni siquiera se conoce con exactitud sus dimensiones, consecuencias, extensión de posibles daños, etc.

Para complejizar aún más la situación, Barros y Camilloni ${ }^{10}$ sostienen que como muchos efectos nocivos del cambio climático son esperables a un futuro (relativamente) lejano, hace que se quite urgencia "a la toma de decisiones en materia de mitigación de las emisiones, por cuanto esta solo será

\footnotetext{
${ }^{6}$ Tenemos a la ley N ${ }^{\circ}$ 26190, del año 2006, titulada: "Régimen de Fomento Nacional para el uso de fuentes renovables de energía destinada a la producción de energía eléctrica".

${ }^{7}$ Mediante decreto del Poder Ejecutivo nacional 891/2016.

${ }^{8}$ Aprobación por ley $\mathrm{N}^{\circ} 27.270$, proponiendo: "mantener el aumento de la temperatura media mundial muy por debajo de 2 ${ }^{\circ} \mathrm{C}$ con respecto a los niveles preindustriales, y proseguir los esfuerzos para limitar ese aumento de la temperatura a $1,5^{\circ} \mathrm{C}$ con respecto a los niveles preindustriales, reconociendo que ello reduciría considerablemente los riesgos y los efectos del cambio climático".

${ }^{9}$ Por resolución 248/2005 de la entonces Secretaría de Ambiente y Desarrollo Sustentable (actual Ministerio).

${ }^{10}$ Expertos del CONICET en temáticas de cambio climático.
} 
efectiva más allá de la mitad del siglo, y resulta muy difícil para cualquier sociedad admitir sacrificios en beneficio de las generaciones futuras" (2016: 60).

En relación a la incertidumbre que significa el cambio climático para los Estados, Beck sostiene que los Estados (de bienestar) tradicionalmente gestionaron riesgos sobre los cuales puede hacerse cierto cálculo de previsibilidad, como ser "la creación de los esquemas de compensación y seguro para accidentes y lesiones en el trabajo y para el desempleo" (1998: 508). Sin embargo, frente a los riesgos creados por el cambio climático y sus características de incalculables e inciertos, dichas formas tradicionales de administrarlos y compensarlos, ya no funcionan. No es comparable un riesgo de desempleo o accidentes laborales, que riesgos derivados de posibles catástrofes climáticas, en cualquier lugar y en cualquier momento.

\section{1. "Crisis de la ciencia"}

Debemos agregar otro desafío para los gestores de políticas públicas (quizás, uno de los mayores), el cual podríamos denominar como "la crisis de la ciencia" en un doble sentido. En primer lugar, debido a que, como ya se señaló, más conocimiento técnico-científico no implica mayor control de los riesgos, sino todo lo contrario ${ }^{11}$. En un segundo sentido, y es el que se quiere resaltar en este apartado, se habla de "crisis de la ciencia" en tanto que la construcción y dotación de significado del término "riesgo" ya no es una tarea exclusivamente de los expertos (científicos), sino que la sociedad en general, al sentirse amenazada, se introduce de lleno en la disputa por la construcción del concepto de riesgo ${ }^{12}$.

En este sentido, Ciselli (2011:4) propone que "construimos determinadas categorías culturales a partir de ciertas posiciones sociales, por lo tanto la noción de riesgo es una noción construida culturalmente que enfatiza algunos aspectos del peligro y que descarta otros", asimismo "dado el carácter de categoría social que tiene la noción de riesgo, respecto a su aceptabilidad nadie es un experto sino que la construcción del mismo depende de la percepción cultural de los peligros" (2011: 5). Es decir, que en sintonía con lo propuesto por Ciselli, se puede pensar que el Estado argentino se ve ante la urgencia de diseñar políticas públicas contra el cambio climático no solamente por recomendación de la ciencia, sino porque la interacción social y cultural del país ha llevado a visibilizar ciertos peligros y amenazas, entre las que se encuentran los desastres meteorológicos como inundaciones (en ciudades y en zonas rurales), sequías, derretimiento de glaciares, olas de calor, etc., atribuidos en mayor medida al fenómeno del cambio climático. Y considerando los aportes de Beck ${ }^{13}$ y Giddens, podemos pensar que la sociedad argentina universalizada, destradicionalizada y reflexiva, percibe culturalmente al cambio climático como un riesgo actual e inminente.

\section{En sintonía con la propuesta de Ciselli, Beck sostiene que los}

riesgos que se generan en el nivel más avanzado del desarrollo de las fuerzas productivas (ej. radiactividad) [...] se basan en interpretaciones causales, por lo que sólo se establecen en el saber (científico o anticientífico) de ellos, y en el saber pueden ser transformados, ampliados o reducidos, dramatizados o minimizados, por lo que están abiertos en una medida especial a los procesos sociales de definición. Con ello, los medios y las posiciones de la definición del riesgo se convierten en posiciones sociopolíticas clave (2006: 33-34).

\footnotetext{
${ }^{11}$ Ver apartado 1, p. 5 de esta ponencia.

12 A los fines de facilitar la claridad se contrasta entre saber científico y saber social, pero el panorama es de mayor complejidad, teniendo en cuenta que dentro de cada saber o racionalidad, existen asimismo disputas y contradicciones. En el saber científico se plantean siempre "aseveraciones y perspectivas rivales y contradictorias procedentes de una variedad de actores y grupos afectados que definen el riesgo de formas muy distintas" (Wynne citado en Beck, 1998: 506).

${ }^{13}$ Beck explica que "las constataciones del riesgo son una simbiosis aún desconocida, no desarrollada, entre ciencias de la naturaleza y ciencias del espíritu, entre racionalidad cotidiana y racionalidad de los expertos, entre interés y hecho"; y concluye diciendo que "[las constataciones del riesgo] ya no pueden ser aisladas por uno u otro especialista y ser desarrolladas y fijadas de acuerdo con los propios estándares de racionalidad. Presuponen una colaboración más allá de las trincheras de las disciplinas, de los grupos ciudadanos, de las empresas, de la administración y de la política, o (lo cual es más probable) se resquebrajan entre éstas en definiciones opuestas y luchas de definiciones" (2006:42).
} 
De aquí se derivan dos importantes preceptos: en primer lugar, queda claro que los riesgos no son algo dado o estáticos, sino que su configuración además de ser influida por el saber científico, dependen en gran medida de la sociedad, de los interese económicos y de la política que los someten a procesos de definición, percepción y transformación. En segundo lugar, se podría pensar que toda política pública ambiental tendrá como trasfondo una particular elaboración y concepción propia del riesgo que quiere gestionar. Con lo cual, queda en evidencia la trascendencia que tiene el poder dominar y controlar el proceso social de definición de los riesgos ambientales, ya que del resultado de dicho proceso, se diseñarán y ejecutarán ciertas políticas públicas, en desmedro de otras.

Esta crisis de la ciencia, en su segundo sentido de disputa con otros saberes y prácticas, pone en jaque a los gestores de políticas públicas. Imagínese que el Estado debe decidir sobre la apertura o no de una central nuclear en una población. Aquí se podría pensar qué posición asumiría la racionalidad científica versus la racionalidad social. Por un lado, se acudirá al asesoramiento de expertos, quienes, según Beck se limitarán a valorar "determinados riesgos cuantificables en el caso de accidentes probables"; pero, por otro lado la racionalidad de la sociedad apuntará y denunciará al "potencial de catástrofe de la energía nuclear", ya que "una probabilidad de accidente mantenida en el mínimo es demasiado elevada allí donde un accidente significa el exterminio" (2006: 36).

Del ejemplo dado se infiere que el saber científico puede brindarle a los gestores ciertos datos probabilísticos de posibles riesgos, y como evitarlos, pero la pregunta que la ciencia no puede responder es ¿cuánto riesgo es tolerable por la sociedad? ¿Cuál es el límite de tolerancia acerca de vivir expuestos a riesgos, por más que se mantengan en "los parámetros científicos"? Estas disputas hacen que los políticos ya no puedan acudir únicamente a una fuente de información científica para basar sus decisiones, y deban buscar y encontrar en la sociedad, la legitimación que la ciencia ya no otorga.

\section{Dos posibles soluciones}

Frente al panorama problemático planteado ut supra, y aceptando que la ciencia ya no representa una legitimación directa y exclusiva para tomar decisiones políticas, debemos preguntarnos si existen algunas soluciones o herramientas de la que puedan valerse no solo las políticas públicas ambientales, sino también el derecho ambiental. Se proponen dos: una "ecología democrática" y una profundización del principio precautorio ambiental.

\subsection{Ecología democrática}

Ciselli expresa que:

si bien el discurso científico de los expertos es el que se prioriza al momento de tomar una decisión, resultaría altamente beneficioso dar lugar a otras voces no expertas, a otros discursos sociales que podrían ser oídos en el proceso judicial y por ende a otras esferas del conocimiento. La visibilización del conflicto ambiental, su discusión en diversos espacios sociales, abre la posibilidad a numerosas innovaciones jurídico-institucionales dado que es a partir de las 'crisis, las catástrofes, los accidentes' cuando el campo del derecho se modifica (2011: 7).

En nuestro ordenamiento jurídico ambiental es frecuente la exigencia de realizar audiencias públicas (por ejemplo, en el marco de evaluaciones de impacto ambiental) cuando se quieren emprender obras de relevancia, o introducir cambios en el ambiente. Así, la ley 10208 de la Provincia de Córdoba: "La Autoridad de Aplicación instrumentará como parte integrante de todo procedimiento administrativo de Evaluación de Impacto Ambiental, con carácter obligatorio y previo al otorgamiento o denegatoria de la Licencia Ambiental, audiencias públicas u otros mecanismos que aseguren la participación ciudadana de acuerdo a lo que establece la presente Ley" (artículo 13) (el resaltado me pertenece). 
Queda clara la importancia de incluir y tener en cuenta otros actores y otras visiones del mismo problema ambiental, como el cambio climático. En este mismo sentido, Beck introduce el concepto de democracia ecológica, donde lo ideal sería que "las zonas de decisión deben abrirse al escrutinio y debate públicos. Las decisiones económicas corporativas, las agendas de investigación científica, los planes de desarrollo y el despliegue de nuevas tecnologías deben abrirse todos a un proceso generalizado de discusión y debe desarrollarse un marco legal e institucional para su legitimación democrática" (1998: 513).

Giddens postula que debido a la imponderabilidad de la incertidumbre, las políticas públicas no pueden imponerse "desde arriba" sino que debe existir un compromiso reflexivo con diversos sistemas de la sociedad. Es especialmente interesante la propuesta de Giddens (1997: 25) cuando señala la "necesidad de impulsar formas más radicales de democratización", introduciendo el concepto de democracia dialogante en sintonía con la democracia ecológica de Beck. Explica el autor que cuando en una sociedad los niveles de reflexividad son mayores, es necesario resolver los problemas en un espacio público en el cual realmente se dialogue, dejando de lado las tradicionales formas de legitimidad política basadas, por ejemplo, en simbolismos de poder prestablecidos.

Por último, Giddens señala el rol clave de los movimientos sociales (piénsese en los movimientos ambientalistas) para impulsar la democracia dialogante, logrando "forzar la introducción, en el terreno de debate, de aspectos de la conducta social que anteriormente no tenían discusión, o se 'resolvían' con arreglo a las prácticas tradicionales" (1997:26).

\subsection{Profundización del principio precautorio ambiental}

Como posible solución al dilema de la toma de decisiones de políticas, tiene un fuerte peso en la legislación y -cada vez más- en la jurisprudencia, la aplicación del principio precautorio ambiental, el cual es definido por el art. 4 de la ley nacional 25.675 (Ley General del Ambiente) en los siguientes términos: "Cuando haya peligro de daño grave o irreversible la ausencia de información o certeza científica no deberá utilizarse como razón para postergar la adopción de medidas eficaces, en función de los costos, para impedir la degradación del medio ambiente". La importancia de este principio radica en que se reconoce la posibilidad de que exista incertidumbre científica, y que, pese a ello, el Estado de todos modos deba actuar en pos de la defensa del medio ambiente y la evitación de daños.

El mentado principio tiene además una importancia trascendental, debido a que su correcta aplicación constituye una herramienta jurídica estratégica para evitar daños por riesgos que son, en principio, invisibles a los sentidos. Recordemos que uno de los caracteres de los riesgos que brinda Beck es el de ser muchas veces imperceptibles-invisibles, como podría ser el peligro cancerígeno del contacto con algún agroquímico, aplicado por trabajadores agrarios, o bien ciertas emisiones de químicos que contaminan el aire sin que nos demos cuenta. El principio precautorio ambiental viene precisamente a frenar este tipo de situaciones, y debe ser utilizado para tomar medidas que eviten daños a la población y al ambiente, por más que los peligros estén rodeados de un halo de incertidumbre o invisibilidad en los términos de Beck.

En relación a los riesgos que implica el cambio climático, se sostiene que el principio precautorio ambiental justifica y legitima las políticas públicas para luchar contra el mismo, por más que haya ciertas voces en contra y que dudan de su real existencia y causas antrópicas.

En último lugar, cabe reflexionar sobre qué otro rol puede desempeñar el derecho ambiental ante los riesgos de la modernidad: además de la función precautoria y de prevención, Beck señala la necesidad de "redefinir los estándares (de responsabilidad, seguridad, control, limitación de daños y distribución de las consecuencias del daño)" (1997: 19-20). Es importante que el derecho ambiental tenga en cuenta a la realidad amenazante y futura ante la cual nos encontramos, y regule conforme a ella, limitando los posibles daños y consecuencias irreversibles, y brindando herramientas efectivas de control y seguridad. 


\section{Reflexiones finales}

Podríamos decir que frente al socavamiento de la legitimidad de la ciencia para fundar decisiones políticas, se podrían impulsar procesos que profundicen a la democracia, o en otras palabras, salvando la redundancia, impulsar "la democratización de la democracia". En este sentido, se considera que en la política ambiental argentina están dadas las bases jurídicas-normativas para comenzar dicho proceso de profundización, ya que tenemos una legislación ambiental completa y extensa, donde se recepta el ya mencionado principio precautorio, y donde se le da mucha relevancia a las audiencias públicas y evaluaciones de impacto ambiental como elementos previos y necesarios para fundar decisiones de política ambiental.

Pero, pareciera que no alcanza con contemplar y receptar jurídicamente dichos elementos, sino que es necesario y urgente adoptar dos tipos de medidas: por un lado, dar un impulso y reforma institucional que permita acercar a la ciudadanía las herramientas de información y participación ambientales, y por otro lado, educar y fomentar para que las personas y movimientos sociales ejerzan su derecho de participar en el terreno de las decisiones políticas frente al cambio climático. Ya que sin instituciones preparadas y sin una ciudadanía participativa que dialogue y dispute los límites de riesgos tolerables, la democracia ecológica de Beck no es más que una utopía.

Por último, y en relación a las políticas públicas contra el cambio climático, se destaca que el carácter a futuro de los riesgos y la voluntad de evitarlos, constituyen el motor y la esencia de las políticas públicas contra el cambio climático, principalmente las políticas de mitigación y adaptación.

\section{Bibliografía}

Barros, V. y Camilloni, I. (2016). La Argentina y el cambio climático: de la física a la política. Buenos Aires: Eudeba.

Beck, U. (2006). La sociedad del riesgo: hacia una nueva modernidad. Barcelona: Paidós. (1997). La política de la sociedad de riesgo. Oxford: Oxford Polity Press.

Beck, U., Giddens, A. y Lash, S. (1997). Modernización reflexiva: política, tradición y estética en el orden social moderno. Madrid: Alianza Editorial.

Beriain, J. (1996). El doble 'sentido' de las consecuencias perversas de la modernidad. En Beriain J. (comp.). Las consecuencias perversas de la modernidad: modernidad, contingencia y riesgo (7-29). Barcelona: Anthropos.

Ciselli, G. (2012). Una reflexión sobre la governancia de los riesgos ambientales: El caso de la mega minería en Esquel (Chubut). Revista Hermeneutic, 11, 1-13

Fresneda, C. (2016, 9 de Septiembre). Cambio climático: 'un cuento chino'. El mundo. Recuperado de http://www.elmundo.es/internacional/2016/11/09/58236dede2704ebc6a8b4575.html

Giddens, A. (1998). Introducción. En Más allá de la izquierda y la derecha (11-30). Madrid: Catedra.

------------. (1998). Política generativa y bienestar positivo. En Más allá de la izquierda y la derecha (157-179). Madrid: Cátedra.

Grupo Intergubernamental de Expertos sobre el Cambio Climático. (2014). Cambio climático 2014: Informe de síntesis. Ginebra: PNUMA. Recuperado de: http://www.ipcc.ch/pdf/assessmentreport/ar5/syr/SYR_AR5_FINAL_full_es.pdf 
Ley de Aprobación de la Convención Marco de las Naciones Unidas sobre el Cambio Climático, $\mathrm{N}^{\circ}$ 24295 (1993)

Ley de Aprobación del Acuerdo de París hecho en París el 12 de Diciembre de 2015 sobre Cambio Climático, $\mathrm{N}^{\circ} 27270$ (2016)

Ley de Política Ambiental Provincial (Córdoba), № 10208 (2014)

Ley de Régimen de Fomento Nacional para el Uso de Fuentes Renovables de Energía Destinada a la Producción de Energía Eléctrica, Nº 26190 (2006)

Ley General del Ambiente, $N^{\circ} 25675$ (2002).

Secretaría de Ambiente y Desarrollo Sustentable de la Nación. (2015). Tercera Comunicación Nacional de la República Argentina a la Convención Marco de las Naciones Unidas sobre el Cambio Climático. Buenos Aires: Secretaría de Ambiente y Desarrollo Sustentable de la Nación. Recuperado de: http://www.msal.gob.ar/salud-y-desastres/index.php/riesgos-de-desastres-en-argentina/principalesamenazas/ola-de-calor 